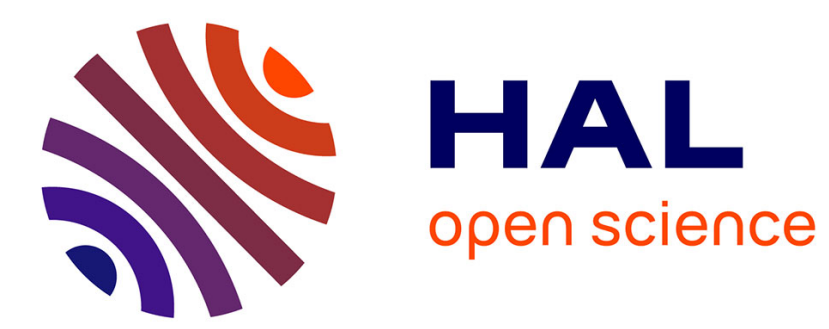

\title{
Forecast scheduling for mobile users
}

\author{
Hind Zaaraoui, Zwi Altman, Eitan Altman, Tania Jimenez
}

\section{To cite this version:}

Hind Zaaraoui, Zwi Altman, Eitan Altman, Tania Jimenez. Forecast scheduling for mobile users. 27th IEEE International Symposium on Personal, Indoor and Mobile Radio Commuinications (PIMRC'16), Sep 2016, Valence, Spain. hal-01332507

\section{HAL Id: hal-01332507 https://hal.science/hal-01332507}

Submitted on 16 Jun 2016

HAL is a multi-disciplinary open access archive for the deposit and dissemination of scientific research documents, whether they are published or not. The documents may come from teaching and research institutions in France or abroad, or from public or private research centers.
L'archive ouverte pluridisciplinaire HAL, est destinée au dépôt et à la diffusion de documents scientifiques de niveau recherche, publiés ou non, émanant des établissements d'enseignement et de recherche français ou étrangers, des laboratoires publics ou privés. 


\title{
Forecast scheduling for mobile users
}

\author{
Hind Zaaraoui*, Zwi Altman*, Eitan Altman ${ }^{\dagger}$ and Tania Jimenez ${ }^{\ddagger}$ \\ * Orange Labs \\ 38/40 rue du Général Leclerc,92794 Issy-les-Moulineaux \\ Email:\{hind.zaaraoui,zwi.altman\}@orange.com \\ ${ }^{\dagger}$ INRIA Sophia Antipolis and LINCS, France \\ Email:Eitan.Altman@sophia.inria.fr \\ $\ddagger$ Avignon University \\ Email:tania.jimenez@univ-avignon.fr
}

\begin{abstract}
In future networks, Radio Resource Management (RRM) could benefit from Geo-Localized Measurements (GLM) thanks to the Minimization of Drive Testing (MDT) feature introduced in Long Term Evolution (LTE). Such measurements can be processed by the network and be used to optimize its performance. The purpose of this paper $^{\mathrm{a}}$ is to use GLM to significantly improve scheduling. We introduce the concept of forecast scheduler for users in high mobility that exploit GLM. It is assumed that a Radio Environment Map (REM) can provide interpolated Signal to Interference plus Noise Ratio (SINR) values along the user trajectories. The diversity in the mean SINR values of the users during a time interval of several seconds allows to achieve a significant performance gain. The forecast scheduling is formulated as a convex optimization problem namely the maximization of an $\alpha$-fair utility function of the cumulated rates of the users along their trajectories. Numerical results for thee different mobility scenarios illustrate the important performance gain achievable by the forecast scheduler.

Index Terms-Forecast scheduler, alfa-fair, high mobility, Minimizing Drive Tests, MDT, Radio Environment Maps, REM, geolocalized measurements
\end{abstract}

\section{INTRODUCTION}

The use of GLM for the optimization and troubleshooting of the Radio Access Network (RAN) is one of the challenging topics in future RANs. The feature that allows to perform such measurements has been introduced into the LTE standard under the term MDT. The term MDT was motivated by the need to replace costly drive tests needed to manage and to troubleshoot the network by mobile based automatic GLM. However, as is shown in this paper, GLM has the potential to be used not just for troubleshooting the RAN but for designing powerful RRM algorithms that can considerably improve the network performance.

MDT measurements can be performed in immediate or idle mode [1]. In immediate mode a connected mobile performs the GLM and immediately reports them to the Base Station (BS). In idle mode the mobile performs the measurements according to the predefined configuration (storing periodicity, logging duration, etc, and reports them to the BS once it enters a connected mode. The MDT feature is presently available in mobile chipsets but not yet activated.

The perspective of having GLM has opened an active research and development domain, namely the construction

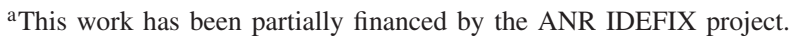

of a REM using spatial interpolation techniques ([2], [3]). The REM can be created and updated in a MDT server in the management plane and be downloaded into each BS (see Fig.1). It can provide maps for different quantities such as the received signal strength or SINR. The BS can then use the REM to optimize RRM algorithms e.g. for association, handover, or resource allocation.

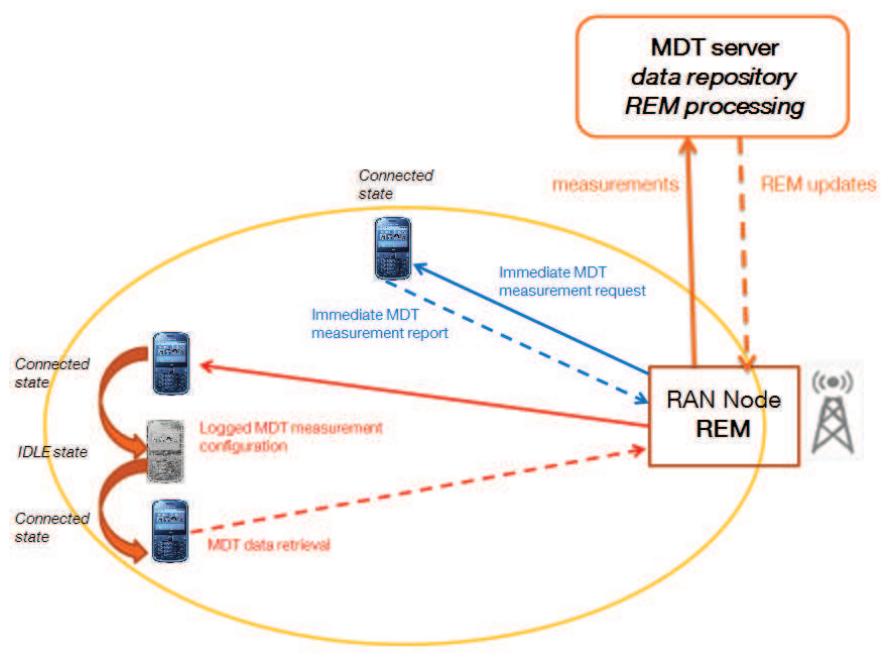

Fig. 1. MDT data collection and coverage prediction

In this paper we introduce the concept of Forecast Scheduling which is a scheduling model for users in high mobility that uses GLM. Vehicular users are considered, and it is assumed that by means of a Global Positioning System (GPS) the users can report their location and speed to the BS. It is further assumed that during a time window $T$ of the order of seconds, the BS can predict the SINR and data-rate variations of the users along their trajectories, e.g. using a REM. Figure 2 depicts the trajectories of three users with the corresponding data-rates. Various techniques for vehicle speed modelling and prediction have been developed, such as one related to the ARIMA model [4]; non-parametric regression [5]; Kalman filtering model [6]; or Neural Network [7].

In public transport (bus, tramway, train...), highways and trunk roads, the users trajectories are well known and can be considered as deterministic. However, trajectories may not 
always be predictable, e.g. in urban environment. Previous works (e.g. [8]) developed trajectory prediction in order to manage the handoff and rerouting of connection problems. The uncertainty in the trajectory prediction can be reduced by decreasing the time window $T$, according to the environment. Interestingly, it is shown that even for a time window of two seconds, the forecast scheduler can achieve significant performance gain.

The forecast scheduler allocation during a time window $T$ is formulated as a convex optimization problem, namely the maximization of an $\alpha$-fair utility function of the cumulated rates of the mobile users along their trajectories. Similarly to the classical $\alpha$-fair scheduling such as the Proportional Fair (PF) [9], the forecast scheduler is an opportunistic scheduler with a degree of fairness depending on the choice of the $\alpha$-fair parameter. However, the scheduling gain is not related to the user diversity in fast fading states (measured in a millisecond timescale) as in classical $\alpha$-fair scheduler. In fact, vehicular users moving with speeds of $30 \mathrm{~km} / \mathrm{h}$ and above experience a too short coherence time to benefit from fast fading. The forecast scheduling gain is due to the diversity in the average SINR which is known in advance at any given time along the users' trajectories.

The difference between the SINR predictions and the actual values is referred here as an error, and its estimation is addressed in the paper. This error may distort the forecast scheduling strategy and impact the network performances. In this paper we consider the errors due to interference variations.

The idea of improving resource allocation by anticipating the future state of the mobile user has recently became an active research arena, mainly for video streaming services, see for example [10] and [11]. This research area has been denoted as anticipatory or proactive resource allocation. [12] for example develops a formulation based on Markov Decision Process (MDP) that allocates higher rates when channel deterioration is anticipated. The present paper considers elastic type of traffic and proposes a solution that integrates any desired degree of fairness via the $\alpha$-fair formulation. Furthermore, to our knowledge, this is the first contribution that investigates the utilization of a REM in the design of an advanced scheduler.

The paper is organized as follows. Section II describes briefly the system model. Section III presents the forecast scheduling model and its formulation as a convex optimization problem. A methodology for evaluating the impact of SINR prediction errors is addressed in Section IV. Numerical results for the evaluation of the performance of the forecast scheduler are presented in Section $\mathrm{V}$ followed by concluding remarks in Section VI.

\section{SYSTEM MODEL}

Consider an omni-directional macro-cell (BS) surrounded by six interfering neighbouring macro-cells. A Virtual Small Cell (VSC) is deployed close to the border of the cell (see Figure 3a). A VSC (also denoted as Virtual Sectorization $(\mathrm{ViS}))$ is a remotely created small cell using an antenna array that radiates a narrow beam, and can be installed beside the macro-cell antenna [13]. The purpose of considering a VSC is to create a limited area in the cell in which important SINR variations can be experienced. A single scheduler allocates resources to the macro-cell and the VSC which share the same frequency bandwidth (with no frequency reuse).

A REM is deployed in the BS and provides SINR values corresponding to the mobile location. Vehicular mobile users with a fixed speed of $50 \mathrm{~km} / \mathrm{h}$ are considered along one or two trajectories. The spatial resolution of the REM is of $1 \mathrm{~m}$ (it is recalled that the REM interpolates GLM), and in $50 \mathrm{~km} / \mathrm{h}$ it corresponds to a $70 \mathrm{~ms}$ time intervals over which the SINR is considered constant. Hence the time resolution of the forecast scheduling is of $70 \mathrm{~ms}$. During this time interval, a fixed allocation is applied, namely the same users are scheduled at a rate depending on the technology (e.g. $1 \mathrm{~ms}$ for LTE.

The data rates are calculated from the SINR values using the Shannon formula:

$$
\phi(S I N R)=W \log _{2}(1+S I N R),
$$

where $W$ is the bandwidth.

We suppose that there are no arrivals or departures of users during the scheduling duration. Full buffer users are assumed, namely having an infinite volume to transmit. The generalization of the model to account for arrivals and departures is addressed in the next section.

\section{FORECAST SCHEDULING MODEL}

Consider $n$ users moving at a constant speed during a time interval $T$ - the scheduling period, over which $n$ is considered constant. Suppose that time is in discrete space: $t \in\{1,2, . ., T\}=[|1, T|]$ and $i$ denotes the user number, $i \in\{1,2, . ., n\}=[|1, n|]$. During a scheduling period denoted here for simplicity as a unit time (e.g. 70ms), the bandwidth is shared among the scheduled users. Let $a_{i}$ denote the bandwidth proportion allocated to a user $i, a_{i}(t) \in[0,1]$, according to the scheduling strategy, and $W$ - the total bandwidth. Then the throughput of user $i$ is given by

$$
a_{i} \phi\left(S I N R_{i}\right)=a_{i} W \log _{2}\left(1+S I N R_{i}\right)
$$

Denote by $\phi\left(S_{i}^{t}\right)$ the throughput of user $i$ at time $t$ with $S_{i}^{t}$ being the predicted SINR. Denote by $a_{i}(t)$ as the scheduling allocation of the user $i$ at time $t$ where $\forall t, \sum_{i=1}^{n} a_{i}(t)=1$.

The forecast scheduling allocation policy is defined by the following optimization problem, with $\alpha \neq 1$ :

$$
\begin{array}{r}
\text { maximize: } f(a)=\sum_{i=1}^{n} \frac{\left(\sum_{t=1}^{T} a_{i}(t) \phi\left(S_{i}^{t}\right)\right)^{1-\alpha}}{1-\alpha} \\
\text { with }: \forall i, \forall t, a_{i}(t) \geq 0 \\
\text { and }: \forall t, \sum_{i=1}^{n} a_{i}(t)=1
\end{array}
$$




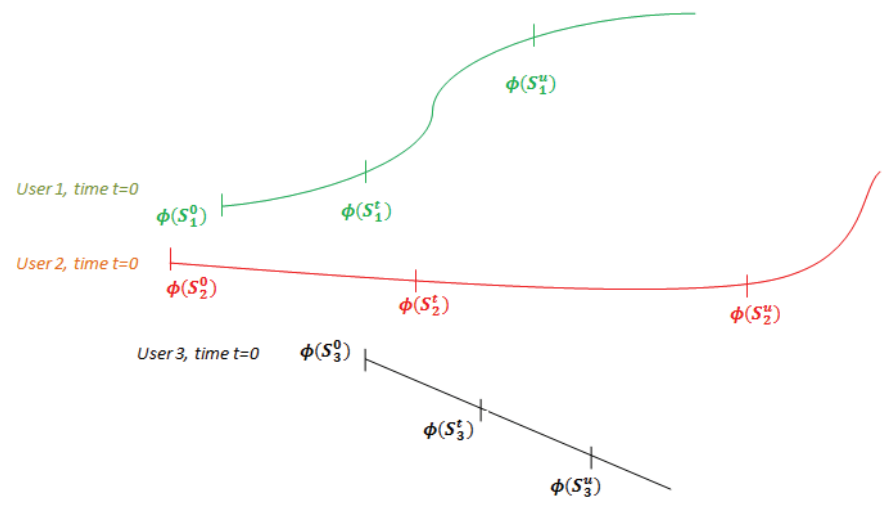

Fig. 2. Example of 3 users with 3 different predictable trajectories. $\phi\left(S_{i}^{t}\right)$ is the predicted data-rate of user $i$ at time $t$.

For $\alpha \rightarrow 1$, the optimization problem with the same constraints reads:

$$
\text { maximize : } f(a)=\sum_{i=1}^{n} \log \left(\sum_{t=1}^{T} a_{i}(t) \phi\left(S_{i}^{t}\right)\right) \text {. }
$$

Both problems (3) and (4) are convex, and can be solved using powerful convex optimization solvers, e.g. CVX (see Section V for more details). The size of the optimization problem is defined by the number of unknown variables, namely $n \times T$.

The formula (4) is used in this study. The interpretation of (4) is the following: resources are shared fairly among the users according to the data-rate variation in their future trajectories. For example, if a user has a coverage hole in his future trajectory, the forecast scheduler will take this into account and allocate this user as much data as possible before reaching the coverage hole while remaining fair with respect to the other users. Detailed examples are given in Section V.

In case the number of users during the interval $T$ changes due to a new arrival or departure, the forecast scheduler could start from the beginning. However certain users may have not yet been scheduled during $T$, causing a problem in the scheduler optimality. One can envisage simple heuristic approaches for handling arrival and departures. For example, the time slots scheduled for a user that leaves the network can be allocated in a Round Robin (RR) fashion to the remaining users.

One can let the number of users vary during time but in this case the function $f$ in (3) should be modified to take into account a stopping time $\tau$, namely the time when the users' number varies. Denote by $f_{t}$ the new function to maximize in the case where users' number can vary. The constraints remain the same as in (3):

$$
f_{t}(a)=\mathbb{E}\left(\sum_{i=1}^{n(t)} \frac{\left(\sum_{j=t}^{t+\tau} a_{i}(t) \phi\left(S_{i}^{t}\right)\right)^{1-\alpha}}{1-\alpha}\right),
$$

where $\tau=\inf \{j>t, n(j)-n(j-1) \neq 0\}$, and $n(t)$ is the number of users in the cell at time $t . \tau$ is measured from time $t$. The expectation is calculated for the variable $\tau$. The rational of (5) is to maximize $f_{t}$ as long as the number of users is fixed.

\section{SINR PREDICTION ERROR}

The forecast scheduler utilizes SINR values provided by a REM. These values are averaged and depend on the interference level during the measurement time, which depend on the loads of the interfering cells. We expect that the dominant contribution to the difference between the actual SINR experienced by the user and the predicted value is interference. This difference is denoted as the prediction error. Example of other sources of errors include fast fading, and interpolated errors of the REM. We consider here only the error due to interference.

In order to evaluate the impact of the prediction error on the performance of the forecast scheduler, we assume an extreme (pessimistic) case: The REM provides SINR predictions for an overloaded network, i.e. the interfering cell have maximum load that equals 1 . The forecast scheduler performance will be compared with that of a network with low mean load value, and is denoted as actual network. The interference in the actual network varies in time and its impact is calculated in two different ways. In the first, dynamic interference fluctuations is simulated at each scheduling iteration. In the second, the mean interference fluctuation is calculated together with the mean distance between $E(I)$ or $\max (I)$ and the random variable $I$ as is explained presently.

Interference modeling: We assume elastic traffic model in which all the BS resources are allocated, even if there is just one user. A any time, a neighboring cell can have one of two states: it can be empty and produce no interference, or it has at least one user and produce maximum interference. The interference term is therefore described as follows:

$$
I=\sum_{i=1}^{n} 1_{\left(N_{i}>0\right)} \frac{P_{i} h_{i}}{r_{i}^{\gamma}}
$$

where $N_{i}$ is the number of users in the neighboring cell $i$, $P_{i}$ - the transmitted power, $h_{i}$ - the channel gain including the path loss and antenna gain, and $r_{i}$ - the distance from the base station $i$ to the desired location. We rewrite $1_{N_{i}>0}=V_{i}$ where $V_{i}$ is a Bernoulli random variable with parameter $p$

$$
I=\sum_{i=1}^{n} V_{i} \frac{P_{i}}{r_{i}^{\gamma}} .
$$

All the $V_{i}$ have the same distribution, but typically they are not independent since each cell interferes with the other ones. However we suppose that $V_{i}$ are independent due to the small impact on the interference estimation. $p$ is defined as the mean load of the cell.

We want to know how the variation of distance $d$ between the mean $E(I)$ or $\max (I)$ and the random variable $I$ impacts the forecast scheduling. We define the distance $d$ as $d(I, E(I))=\sqrt{E\left((I-E(I))^{2}\right)}$, which is the standard 
deviation of $I, \sigma(I)$. We use the following approximation for the SINR taking into account the interference $\sigma(I)$ (if $\left.\frac{\sigma(I)}{E(I)}<1\right)$ :

$$
S I N R=\frac{P h}{E(I)+\sigma(I)} \approx \frac{P h}{E(I)}\left(1-\frac{\sigma(I)}{E(I)}+\frac{\sigma(I)^{2}}{E(I)}\right)
$$

We estimate $\sigma(I)$ empirically as following:

$$
\sigma(I)=\sqrt{\frac{1}{Z} \sum_{z=1}^{Z}\left(I_{z}-E(I)\right)^{2}}
$$

where $Z$ is the number of experiments, $\left\{I_{1}, \ldots I_{Z}\right\}$ - a set of iid experiments. $\frac{\sigma(I)}{E(I)}$ depends on the distances between a location and neighbor BSs.

If we do not have any experience value of $E(I)$ then we replace it by $\max (I)$ where we suppose that all the neighbor cells have at least one user and interfer the principal studied cell:

$S I N R=\frac{P h}{\max (I)-\sigma_{m}(I)} \approx \frac{P h}{\max (I)}\left(1+\frac{\sigma_{m}(I)}{\max (I)}+{\frac{\sigma_{m}(I)}{\max (I)}}^{2}\right)$

where $\sigma_{m}(I)$ empirically as following:

$$
\sigma_{m}(I)=\sqrt{\frac{1}{Z} \sum_{z=1}^{Z}\left(I_{z}-\max (I)\right)^{2}}
$$

By comparing the throughput gain achieved using predicted and actual SINR values, one can estimate the impact of prediction error. Interestingly, one can also compare the scheduler decisions in two cases using the following formula:

$$
\text { error }=\frac{1}{n \times T} \sum_{i \in \text { users }} \sum_{t \in \text { times }} 1_{\left\{\left|a_{i}(t)-a e_{i}(t)\right|>\epsilon\right\}},
$$

where $a$ is the forecast scheduling strategy using the REM, namely the predicted SINR values and ae is the forecast scheduling strategy using measurements from the actual network and $\epsilon$ - the tolerated error. One can observe (see Section V) that the two strategies provide almost identical Mean User Throughput (MUT).

\section{NUMERICAL RESULTS}

\section{A. CVX resolution}

The optimization problem (3) is convex. We choose in this work the CVX library implemented in Matlab to resolve this convex problem (see [14] and [15]). The CVX resolution process verifies the convexity of the problem and solves it using SDPT3 or SeDuMi. SDPT3 is a Matlab implementation of infeasible path-following algorithms for solving conic programming problems whose constraint cone is a product of semidefinite cones. It uses a predictor-corrector primal-dual path-following method with different types of search direction. SeDuMi is a linear/quadratic/semidefinite solver for Matlab and Octave.

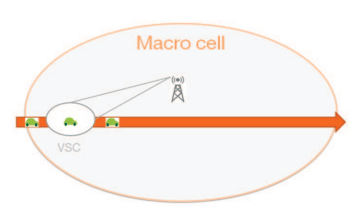

(a) Trajectory with VSC deployment

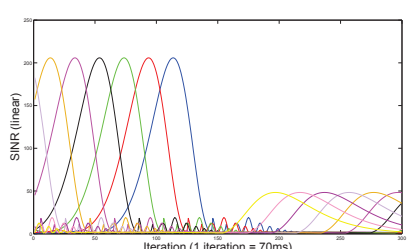

(b) SINR trajectories
Fig. 3. Scenario 1 trajectories

\section{B. Forecast scheduling gain}

Consider mobile users driving at $50 \mathrm{~km} / \mathrm{h}$. At this speed, coherence time is too short to benefit from fast fading diversity and therefore a RR scheduling is used as a baseline. The forecast scheduling model using a REM for a highly loaded network (i.e. for high interference) is compared to the RR baseline scheduler. Simulation parameters including network and mobility parameters are given in Table I.

In order to assess the performance of the forecast scheduler, three scenarios of mobility are considered. They differ from one another by the degree of variation (or smoothness) of the predicted SINR along the mobile trajectories:

- Scenario 1: a VSC (fixed beam) near the cell edge is crossed by a road with vehicular users (Figs.3a and 3b);

- Scenario 2: a road with vehicular users crosses the macro-cell (no VSC, Figs.4a and 4b);

- Scenario 3: two vehicular users are considered; one user drives along a road that traverses a VSC close to the cell edge, and the other user drives on another road without a VSC (Figs.5a and 5b).

TABLE I

NETWORK AND TRAFFIC CHARACTERISTICS

\begin{tabular}{|c|c|}
\hline \multicolumn{2}{|c|}{ Network parameters } \\
\hline Number of macro BSs & 1 \\
\hline Number of interfering BSs & 6 \\
\hline Macro-cell layout & hexagonal omni sector \\
\hline Intersite distance & $500 \mathrm{~m}$ \\
\hline Bandwidth & $20 \mathrm{MHz}$ \\
\hline \multicolumn{2}{|c|}{ Channel characteristics } \\
\hline Thermal noise & $-174 \mathrm{dBm} / \mathrm{Hz}$ \\
\hline Macro Path loss (d in km) & $128.1+37.6 \log _{10}(\mathrm{~d}) \mathrm{dB}$ \\
\hline \multicolumn{2}{|c|}{ Mobility traffic characteristics } \\
\hline User speed & $50 \mathrm{~km} / \mathrm{h}$ \\
\hline Number of users & 10 \\
\hline Time between vehicles & $1.4 \mathrm{~s}$ \\
\hline File size $\sigma$ & full buffer $(\infty)$ \\
\hline Error tolerated $\epsilon$ (eq.12) & 20.05 \\
\hline Forecast scheduling duration $T$ & $70 \mathrm{~ms}$ \\
\hline One iteration = Scheduling delay & $15 \mathrm{iter}$. \\
\hline Average sliding window &
\end{tabular}

Figure 6 presents the mean user throughput gains for the three scenarios achieved by the forecast scheduler with respect to the baseline RR scheduler. As expected, the gain depends on the degree of SINR variation along the trajectories of the different scenarios. The bigger the SINR variations, the higher 


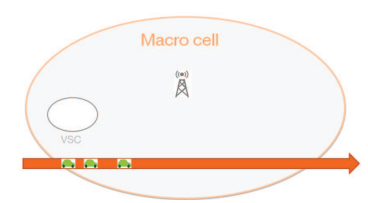

(a) Trajectory without VSC deployment

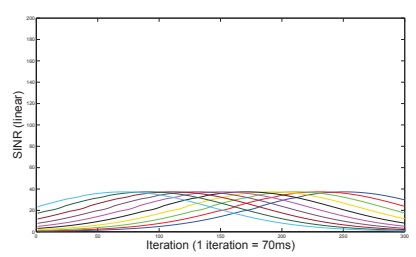

(b) SINR trajectories
Fig. 4. Scenario 2 trajectories

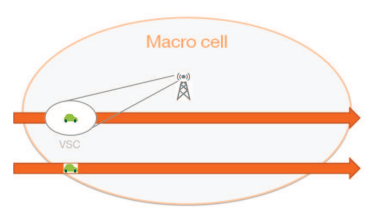

(a) 2 users trajectories

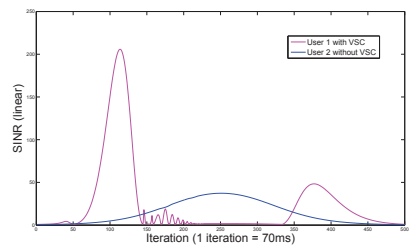

(b) SINR trajectories
Fig. 5. Scenario 3 trajectories

is the forecast scheduling gain. The achieved gains are of $117 \%, 33 \%, 40 \%$ for scenarios 1, 2 and 3 respectively. While the throughput gain for scenario 1 is huge due to the steep SINR variations caused by VSC, it is interesting to see that significant gain can also be achieved for very moderate SINR variations of scenario 2 .

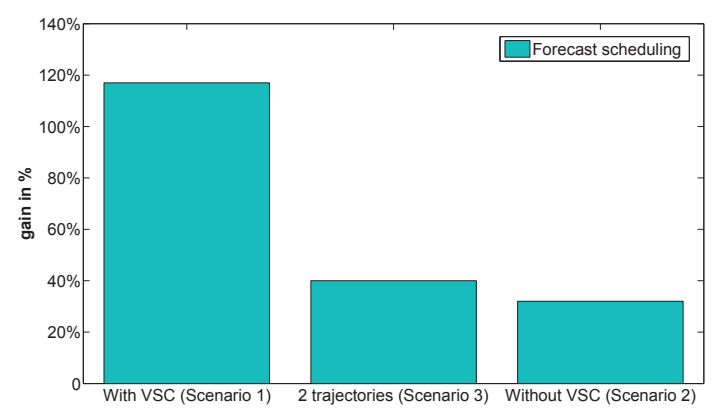

Fig. 6. Forecast scheduling gain compared to the baseline RR scheduling for the three scenarios

\section{Interference error impact}

We investigate the impact of interference on the forecast scheduling for scenarios 1-3 (see Figs.3a-3b to $5 a-5 b$ ). We recall that the REM data corresponds to maximal interference (neighboring cell load of 100\%). We compute the error using eq. (12)) for the three scenarios in the case where the neighbor cells' load is of $10 \%$. In Fig.7, one can notice that the mean variation of the interference (formula (10)) has little impact on the scheduling strategy (red, blue and black charts). However if interference changes randomly in each iteration with average neighbor cells' load of $10 \%$, the error reaches $22 \%$ for the worst case, namely scenario 3 (green curve).
In the case of the mean variation of the interference, a scheduling strategy error of at most $5 \%$ is observed for scenario 1 . The error for scenario 3 reaches $10 \%$ for $T=2 \mathrm{sec}$. For all the scenarios, the error decreases with the increase of the duration $T$ of the forecast scheduling. When the scheduling interval is too short, the SINR dynamics is likely to be limited, and the diversity in the mean SINR along the trajectory can be less exploited. In this case, the impact of interference variations on the scheduler strategy (decisions) will be higher, which explains the increase in the scheduling error for smaller $T$.

Figure 8 shows the forecast scheduling gain in MUT with respect to the baseline RR scheduler for scenario 1 . The gain increases with the scheduling duration $T$. The more SINR information is available in time, the higher is the predictive scheduling gain. One can see that interference error of the REM with respect to the actual network has little impact on the schedular performance measured in terms of mean user throughput.

Figures 9 and 10 depict the normalized throughput variations in time for the three strategies: Forecast scheduling with interference error, forecast scheduling without errors, and the baseline RR scheduling. One can see how the MUT varies dynamically in time for the two extreme cases: $T=2 \mathrm{sec}$ and $T=20 \mathrm{sec}$ for scenario 1 (with a sliding averaging window of $1 \mathrm{sec}=15$ iterations). One can observe that the MUTs for the forecast scheduling with and without interference error are practically the same and are almost always better than the baseline scheduling strategy for both $T=2 s$ and $T=20 \mathrm{~s}$.

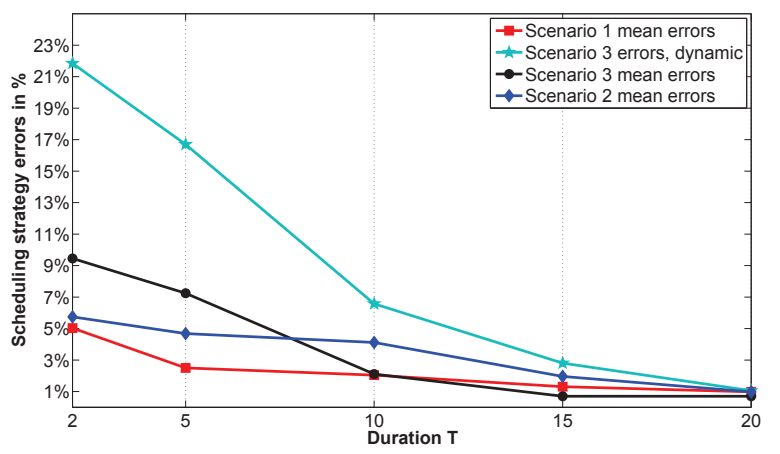

Fig. 7. Forecast scheduling strategy error in $\%$ for the three scenarios and for $10 \%$ mean load of the interfering cells as a function of the scheduling interval $T$.

\section{CONCLUSION}

This paper has introduced the forecast scheduling which is a novel scheduling approach for users in high mobility that utilizes geo-localized measurements. Such measurements can be generated by a REM thanks to MDT data. This scheduling model consists of exploiting predicted SINR variations along the users' trajectories and allocate resources fairly between the users during the scheduling period. The model is based on maximizing a convex utility function under constraints and 


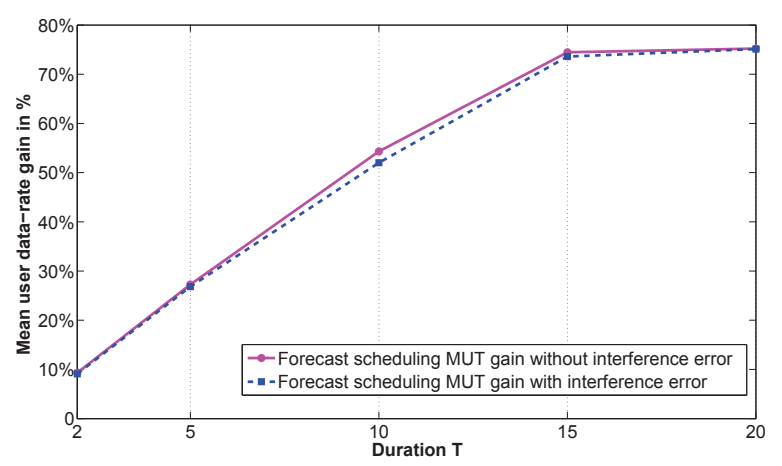

Fig. 8. Forecast scheduling gain in MUT comparing to RR with and without interference error

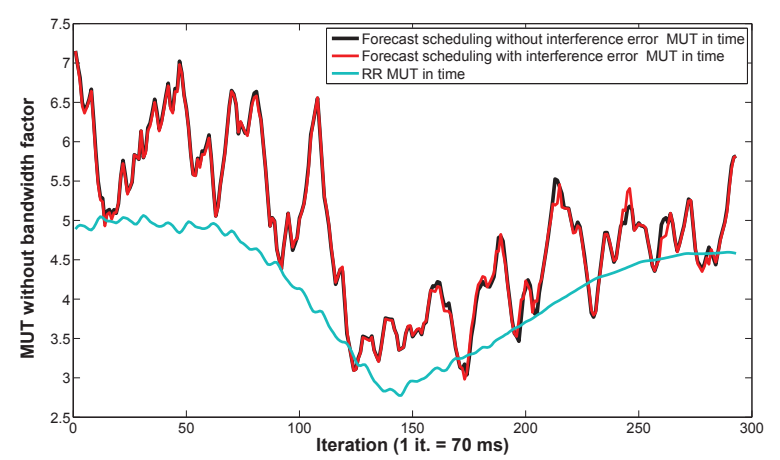

Fig. 9. Time variation of the data-rate for the forecast scheduler with $\mathrm{T}=2 \mathrm{~s}$ and the base line RR scheduler, averaged with 15 iteration sliding window

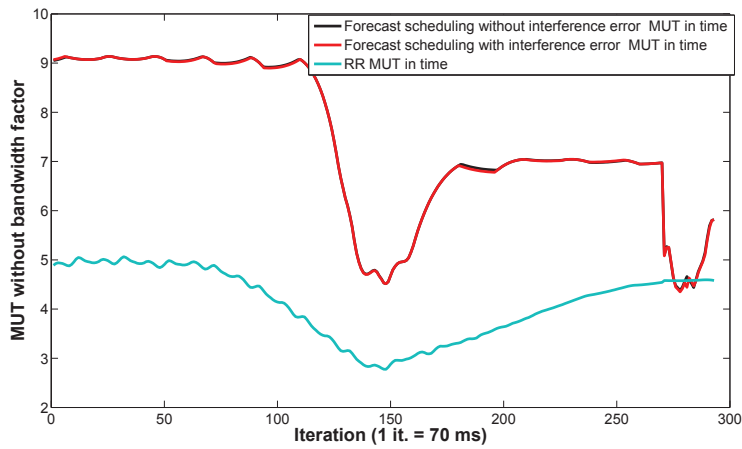

Fig. 10. Time variation of the data-rate for the forecast scheduler with $\mathrm{T}=20 \mathrm{~s}$ and the base line RR scheduler, averaged with 15 iteration sliding window

depends on a $\alpha$ fairness parameter. In this study, the CVX solver has been used to resolve this optimization problem.

We have shown that the forecast scheduling model can achieve very high MUT gains compared to RR scheduling in case of high SINR variations and a long scheduling interval. The gain remains significant also for moderate SINR variations along the trajectory. Simulation scenarios have shown that errors in SINR measurements with respect to the predicted ones (e.g. from a REM) due to interference variations have negligible impact on the obtained throughputs. Such error may distort the forecast scheduling strategy (decisions). This work is just one example of how geo-localised measurements can improve RRM and optimize the network performance. Such measurements are likely to be available thanks to the MDT capable mobiles introduced in $4 \mathrm{G}$ networks.

\section{REFERENCES}

[1] W. Hapsari, A. Umesh, M. Iwamura, M. Tomala, B. Gyula, B. Sebire et al., "Minimization of drive tests solution in 3GPP," IEEE Communications Magazine, vol. 50, no. 6, pp. 28-36, 2012.

[2] H. Braham, S. Ben Jemaa, B. Sayrac, G. Fort, and E. Moulines, "Coverage mapping using spatial interpolation with field measurements," in 2014 IEEE 25th Annual International Symposium on Personal, Indoor, and Mobile Radio Communication (PIMRC). IEEE, 2014, pp. 17431747.

[3] A. Galindo-Serrano et al., "Cellular coverage optimization: A radio environment map for minimization of drive tests," in Cognitive Communication and Cooperative HetNet Coexistence. Springer, 2014, pp. 211-236.

[4] B. M. Williams and L. A. Hoel, "Modeling and forecasting vehicular traffic flow as a seasonal arima process: Theoretical basis and empirical results," Journal of transportation engineering, vol. 129, no. 6, pp. 664672, 2003.

[5] J. You and T. J. Kim, "Empirical analysis of a travel-time forecasting model," Geographical Analysis, vol. 39, no. 4, pp. 397-417, 2007.

[6] Y.-J. Yu and M.-G. Cho, "The system for predicting the traffic flow with the real-time traffic information," Journal of the Korea Institute of Information and Communication Engineering, vol. 10, no. 7, pp. 13121318, 2006.

[7] E.-M. Lee, J.-H. Kim, and W.-S. Yoon, "Traffic speed prediction under weekday, time, and neighboring links' speed: back propagation neural network approach," in Advanced Intelligent Computing Theories and Applications. With Aspects of Theoretical and Methodological Issues. Springer, 2007, pp. 626-635.

[8] T. Liu, P. Bahl, and I. Chlamtac, "Mobility modeling, location tracking, and trajectory prediction in wireless atm networks," IEEE Journal on Selected Areas in Communications, vol. 16, no. 6, pp. 922-936, 1998.

[9] R. Combes, Z. Altman, and E. Altman, "Scheduling gain for frequencyselective Rayleigh-fading channels with application to self-organizing packet scheduling," Performance Evaluation, Feb. 2011.

[10] N. Bui, S. Valentin, and J. Widmer, "Anticipatory quality-resource allocation for multi-user mobile video streaming," in 2015 IEEE Conference on Computer Communications Workshops (INFOCOM WKSHPS). IEEE, 2015, pp. 245-250.

[11] I. Triki, R. El-Azouzi, and M. Haddad, "Anticipating resource management and qoe provisioning for mobile video streaming," arXiv preprint arXiv:1512.05705, 2015

[12] W. Bao and S. Valentin, "Bitrate adaptation for mobile video streaming based on buffer and channel state," in 2015 IEEE International Conference on Communications (ICC). IEEE, 2015, pp. 3076-3081.

[13] A. Tall, Z. Altman, and E. Altman, "Virtual sectorization: design and self-optimization," in 5th International Workshop on Self-Organizing Networks (IWSON 2015), Glasgow, Scotland, May 2015.

[14] I. CVX Research, "CVX: Matlab software for disciplined convex programming, version 2.0," http://cvxr.com/cvx, Aug. 2012.

[15] M. Grant and S. Boyd, "Graph implementations for nonsmooth convex programs," in Recent Advances in Learning and Control, ser. Lecture Notes in Control and Information Sciences, V. Blondel, S. Boyd, and H. Kimura, Eds. Springer-Verlag Limited, 2008, pp. 95-110. 